\title{
Experimental Investigation of Transformations from SBVR Business Vocabularies and Business Rules to Owl 2 Ontologies
}

\author{
Jaroslav Karpovič, Linas Ablonskis, Lina Nemuraitė, Bronius Paradauskas \\ Kaunas University of Technology, Department of Information Systems \\ Studentu st. 50-308, LT-51368 Kaunas, Lithuania \\ e-mail: jaroslav.karpovic@ktu.lt,linas.ablonskis@ktu.lt,lina.nemuraite@ktu.lt,bronius.paradauskas@ktu.lt \\ crossref http://dx.doi.org/10.5755/j01.itc.45.2.8873
}

\begin{abstract}
The goal of the paper is to analyse the relevance of the proposed transformation from business vocabularies and business rules, based on Semantics of Business Vocabulary and Business Rules (SBVR), to the corresponding OWL 2 ontologies. The transformation was aimed to be model driven, lossless and reversible; adaptable to English, Lithuanian and other similar natural languages; covering transformable SBVR concepts and as much as possible OWL 2 concepts, especially those that are important for inference, and supporting the consistency and normalization of resulting ontologies. The paper presents a detailed comparison of the proposed solution with the most advanced, to our knowledge, related works and the experimental investigation of the implemented transformation prototype with nine SBVR business vocabularies and business rule sets with regards to the defined desirable criteria.
\end{abstract}

Keywords: Business concepts; business vocabulary; business rules; representation; meaning; transformation; ontology; SBVR; OWL 2.

\section{Introduction}

The possibility to represent conceptual models and ontologies in human understandable language has attracted the attention of researchers from a long time ago. As the evidence of such a fact, we can mention attempts of verbalizing Entity Relationship, ORM, UML models [1], [2], [3] or development of Controlled Natural Languages [4], [5], [6]. These possibilities became stronger with the appearance of Semantics of Business Vocabulary and Business Rules [7], [8], which has its roots in ORM2 [9], [10] and, by the authors' intent, includes options for representing ontologies (currently, OWL 2 [11]). Several authors have described and implemented transformations from SBVR to OWL 2 [12], [13], [14] (the more exhaustive list of works was analysed in [15]). The purpose of such transformations was not only for business userfriendly creation of ontologies, but also for giving a possibility to check the consistency of SBVR business vocabularies and business rules with ontology reasoners. This is because the SBVR knowledge model until now lacks well-developed analysis tools for reasoning with its complex logics.

We have proposed the SBVR to OWL 2 transformation rules and their implementation prototype, based on some extensions to SBVR metamodel for better usage of the advantages of ontologies. For obtaining consistent ontologies, we have defined several requirements for SBVR business vocabularies and business rules [16], [17], [15]. In particular, our transformation is applied in the semantic search framework, where the user is able to formulate questions in the SBVR structured language. These questions are translated into ontology query language SPARQL and performed in the ontology, obtained from the SBVR business vocabulary and business rules [18]. In such semantic search, the conformance between SBVR business vocabulary, business rules and the ontology concepts has a great importance as well as the possibility to exploit the reasoning features of ontology is desirable.

SBVR and OWL 2 have different metamodels with a part of overlapping concepts, but some part of concepts of both knowledge models has no direct correspondences [8], [11]. The second difficulty in mapping between SBVR and OWL 2 is that there often exist alternative ways for representing the same constructs in both models, so several solutions for transforming SBVR concepts can exist. Therefore, it is difficult to choose the best transformation rules as well as to compare existing solutions. On the base of analysed methodologies for conceptual modelling [10], [19], ontology engineering [20], [21], [22], [23], and our previous works [24], [25], [26], we have 
stated the following criteria for the SBVR to OWL 2 transformation:

1) The transformation should be model driven, i.e., based on metamodels; it should be lossless and reversible for the transformable subset of SBVR concepts;

2) It should cover SBVR concepts that can be transformed into OWL 2 Description Logics (DL) ontologies (we have limited our research to OWL 2 $\mathrm{DL}$ as its reasoning supports the maximum expressiveness while still providing computational completeness and decidability);

3) It should cover ontology concepts allowing using advantages of ontology reasoners for checking consistency of SBVR business vocabularies and business rules as well as for inferring the new knowledge in OWL 2 DL ontologies;

4) It should support the consistency and normalization of resulting ontologies;

5) It should be adaptable to a set of natural languages, e.g., English and Lithuanian, which are not too different.

For reaching the first and second criteria, transformations were defined according to model driven principles and implemented in ATL transformation language [27]; we have developed a solution in order to preserve as much as possible of SBVR semantics in ontology [15]. For the third criterion, we have proposed a few extensions to the SBVR. For supporting the fourth criterion, we have defined requirements for writing SBVR business vocabularies and business rules that allow to obtain consistent and normalized OWL 2 ontologies [17]. It is worth to mention that the third and fourth criteria, to our knowledge, yet have not been deeply examined in related works [12], [13], [14]. The fifth criterion was ensured via language independent transformation rules, which can be adapted to similar languages by setting the defined set of keywords.

The paper presents a comparison of our solution with the most advanced, to our knowledge, SBVR to OWL 2 transformation solutions, and an experimental investigation of the implemented prototype via analysing transformations of nine SBVR business vocabularies and business rule sets in accordance with the presented criteria.

The paper is structured as follows. Section 2 analyses SBVR to OWL 2 transformation implementation methodology and related works. Section 3 presents the comparison of SBVR to OWL 2 transformation solutions. Section 4 presents results of experiment with nine SBVR business vocabularies and business rules, which were transformed into OWL 2 ontologies by our transformation prototype. Finally, Section 5 presents conclusions and future works.

\section{Evaluation of SBVR to OWL 2 transforma- tion: methodology and related works}

The practical principles for ensuring the reversible and lossless transformations were analysed in our previous work [25]. Here we shortly present them. The straightforward way for achieving the lossless bidirectional transformations is to implement transformations in both directions and to test them; however, the rational way is to analyse principles how such transformations could be met. Ehrig et al. [28] presented a formal proof of the sufficient requirements for reversible bidirectional transformations based on graph grammars; their requirements are sufficient to bijective transformations when concepts of two models are mapped 1:1. In practice, the most of required transformations are not bijective. Such a condition also holds for SBVR to OWL 2 transformation. The problem of model driven lossless, reversible and not bijective transformations was analysed by Stevens [29]. She points that no transformation language could guarantee the lossless transformations; the transformations have to be verified. Stevens [29] introduced the concept of the coherent transformation and stated that the coherent transformation is correct, hippocratic and undoable. The correctness of SBVR to OWL 2 transformation means that, for every construct of SBVR metamodel, the direct and reverse transformations should exist. The hipocraticness of transformation means that if transformation is not bijective, then it must not damage the source and target models by rewriting already transformed elements. In our case, the correctness of transformations is ensured by constructing them for all elements of the SBVR that are transformable to OWL 2. The ATL transformation language [27] ensures the hipocraticness of transformations via preventing changes of target constructs obtained during execution of transformations. Undoability means that we should not define transformations that lead to irrevocable changes, i.e., all transformations from the highest to the lowest (e.g., conversion of primitive concepts into OWL 2 data types) should be reversible. The latest requirement is satisfied by construction of transformation rules.

As was concluded in [25], the reversible lossless transformation can be considered as a limited bidirectional transformation that was defined by Hainaut [30]. A transformation $T 1: M \times N \rightarrow N$ is reversible if for all instances $m$ of source models $M$ there exists direct transformation $T 1$ into instances $n$ of target models $N$ and also there exists a reverse transformation $T 2: M \times N \rightarrow M$ such that $\forall m \in M \Rightarrow$ $T 1(m, n)=n, n \in N ; \forall m \in M \Rightarrow T 2(m,(T 1(m, n))=m$, $n \in N$.

Transformation $T 1$ is reversible, but the transformation $T 2$ is not. That means that for transforming the freely chosen OWL 2 ontology into the SBVR business vocabulary and business rules the 
human efforts would be required for supplementing the ontology with appropriate labels [31] as naming of ontology entities can be done by applying different naming rules and it could be impossible to obtain the relevant representations for SBVR concepts. Conversely, the transformation of OWL 2 ontology, obtained by transforming the SBVR business vocabulary and business rules, can be reversible and lossless.

The coverage of OWL 2 ontology concepts for allowing using advantages of ontology reasoners was analysed in our previous works [15]; some extensions to SBVR metamodel for formulating ontology characteristic features, important for inference, were proposed.

The concept of the consistency of the OWL 2 ontology is defined in its direct semantics [32]. Ontology has a vocabulary $V$, defined by the ontology entities (classes, properties, and named individuals), and a set of supported data types called a data type map $D$. Ontology $O$ is consistent in an interpretation $I$, if all axioms in the axiom closure of $O$ are satisfied in $I$. Such interpretation $I$ of ontology $O$ also is a model of $O . O$ is consistent (or satisfiable) w.r.t. $D$ and $V$ if a model of $O$ w.r.t. $D$ and $V$ exists [32].

- The consistency of ontologies is analysed by ontology reasoners according Description Logics (DL) rules. The main functionality of OWL 2 DL reasoners is focused on ([33]):

- Consistency of ontology, i.e., the absence of conflicting facts;

- Concept satisfiability, i.e., the possibility of a class to have any instances;

- Specialization, i.e., computing the subclass relations between every named class to create the complete class hierarchy;

- Realization, i.e., getting all types for a given individual.

In order to ensure the decidability of ontology consistency, concept satisfiability, classification and realization, each class expression with cardinality or ObjectHasSelf restrictions in class expressions of the ontology model can contain only simple object property expressions. Simple object property expressions have no direct or indirect (inherited from property hierarchy) subproperties that are either transitive or are defined by means of property chains [11]. It means that we cannot restrict the cardinality of transitive object properties, etc. Therefore, business rules, whose transformation to OWL 2 results in undesirable expressions, should not be specified in the vocabulary of business rules. However, it is not easy to check such situations in SBVR vocabularies and rules.

Consequently, for checking the consistency of ontology, we need to have an ontology model with the set of individuals sufficient for verifying the satisfiability of all axioms of the ontology. The methodology for engineering ontologies, normalization of ontology schemas and checking their consistency, as well as estimation of the required number of individuals for that purpose, was analysed in our previous work [26], which in turn is based on the works of eminent researchers in the field of ontology engineering. Shortly, we need to have a sufficient number of individuals for each primitive (non-inferable) class in order to compute all inferable classifications; a sufficient number of assertions for each property, which can be possessed by corresponding individuals, and a sufficient number of data values for each data property for computing all restrictions, classifications and realizations. The formal approach for determining the required number of elements is to apply formal concept analysis by creating a lattice of concepts for dependencies among ontology concepts (classes, properties, property characteristics, axioms, and restrictions) [26]. In our case, for checking the consistency of business vocabulary and business rules, the required individuals and assertions can be obtained from the individual concepts and facts specified in the business vocabulary under consideration, or created in ontology obtained after transformation.

We have compared our transformation with the most advanced, to our knowledge, works for transforming SBVR to OWL 2, presented in Kendall and Linehan [12], and Reynares et al. [13]. The first of them [12] proposed a mapping of SBVR vocabularies to a combination of OWL 2 elements and annotations. The authors' goal was to define a reversible mapping from SBVR vocabulary to OWL 2 and back without loss of semantic information though format, syntactic structure, and lexical details may be different. The purpose of such mapping was to offer a format for exchanging SBVR vocabularies between SBVR tools as an alternative to XMI. They gave a mapping of SBVR Date Time concepts [35] (SBVR extensions) to OWL 2 data types, vocabulary captions, and representations, including synonyms and synonymous forms, to annotations. Kendall and Linehan have not considered categorization schemes, segmentations, and reflected only a small subset of business rules; some of their proposed mappings are incorrect [15]. The purpose of Reynares et al. [13] transformations is closer to our proposal; they did not pay any attention to synonymous SBVR representations but tried to cover the greater part of SBVR concepts and logical formulations. However, their transformations seem immature and some of them are incorrect [15].

The SBVR to OWL2 transformation, the $1^{\text {st }}$ version of which was proposed in [16], [17], also is focused on the SBVR meaning, though we capture the primary representation of every concept in ontology labels, and one synonymous form for each verb concept wording, which is needed for representing the inverse object property in the OWL 2 . Annotation labels are conventional means for expressing naming of classes, object and data properties, and individuals. However, they are insufficient for presenting linguistic knowledge extracted from SBVR vocabularies and 
rules. The possibilities to map SBVR terminological and linguistic information were analysed in [15] where we have made the decision to map SBVR business vocabularies and business rules to separate OWL 2 ontologies: domain ontologies for meanings and lexical ontologies for representations. Creation of lexical ontologies from SBVR currently is under development [34] and lies beyond the scope of this paper. Domain ontologies capture primary representation forms for each SBVR concept; one synonymous form can be indicated as an inverse form of each SBVR verb concept for representing inverse object properties in OWL 2.

For transforming SBVR into OWL 2, we have chosen the OWL 2 metamodel based on its direct semantics [32] for its conceptual clarity; the results of transformations are represented in OWL 2 Functional style syntax, which can be converted to other OWL 2 formats (e.g., RDF) [39], [40], [41]. Our transformations are limited to Description Logics, but we do not consider this as a shortcoming as current ontology reasoners are able to work with OWL 2 DL compatible ontologies only. Therefore, SBVR vocabularies under transformation should conform to Description Logics as well as to regard particularities of OWL 2 ontologies allowing efficient reasoning, maintenance and evolution [17].

SBVR to OWL 2 transformation tool prototype was developed in Eclipse environment using ATL and XSLT transformation engines; it can be used as standalone component using SBVR business vocabulary and business rules as input [36], or integrated with SBVR Structured Language Editor (SBVR SLE) [37].

\section{Comparison of SBVR to OWL 2 transformation solutions}

This section presents the comparison of SBVR to OWL 2 transformations defined in this research and related works. The comparison was made on the base of analysed publications (to our knowledge, the implementations of related works are not publicly available or reported). The main peculiarity of our SBVR to OWL 2 transformations is in the fact that we analysed SBVR and OWL 2 knowledge models from three perspectives: 1) what SBVR concepts can be represented in OWL 2; 2) how to represent OWL 2 concepts in SBVR in order to obtain consistent and meaningful ontologies, capable to make inference; 3 ) what is possible and the most appropriate to implement from the research point of view. The demonstrative prototype of our implementation is available at http://s2o.isd.ktu.lt/ [36].

\subsection{Transformation of SBVR meanings}

Table 1 presents a comparison of the most advanced SBVR 1.2 to OWL 2 transformations proposed in publications of different authors (transformations of Reynares et al. are dedicated to SBVR 1.0 [13]).

Table 1. Comparison of SBVR to OWL 2 transformation solutions presented in publications of different authors (the symbols " " mean incomplete mappings, which are explained in references below the table with corresponding references, e.g., $(* i)$

\begin{tabular}{|c|c|c|c|c|}
\hline SBVR 1.2 concepts & Our work [15] & $\begin{array}{l}\text { SBVR } 1.2 \\
{[8]}\end{array}$ & $\begin{array}{l}\text { Kendall and } \\
\text { Linehan [12] }\end{array}$ & $\begin{array}{c}\text { Reynares et } \\
\text { al. (SBVR } \\
1.0[13])\end{array}$ \\
\hline $\begin{array}{l}\text { vocabulary; vocabulary namespace; } \\
\text { namespace URI; language }\end{array}$ & $\begin{array}{l}\text { Ontology; ontology namespace; ontology IRI; } \\
\text { language for annotation properties "label", } \\
\text { "label_sbvr" }\end{array}$ & - & + & - \\
\hline included vocabulary & Imported Ontology & - & $+(* 1)$ & + \\
\hline general concept & Class & + & $\sim(* 2)$ & + \\
\hline role (together with property association) & $\begin{array}{l}\text { Data Property, } \\
\text { Data Property Range }\end{array}$ & - & $+(* 1)$ & - \\
\hline $\begin{array}{l}\text { verb concept role (supplemented with } \\
\text { definition for derivation of individuals, } \\
\text { playing the role) }\end{array}$ & $\begin{array}{l}\text { SubClassOf of class "Role", Equivalent Classes, } \\
\text { Axiom for derivation of Individuals, playing the Role }\end{array}$ & $\sim(* 3)$ & $+(* 1)$ & $\sim(* 4)$ \\
\hline individual concept + classification & $\begin{array}{l}\text { Named Individual + Class Assertion (the type of the } \\
\text { Individual) }\end{array}$ & + & + & + \\
\hline number & decimal & - & + & - \\
\hline $\begin{array}{l}\text { integer; nonnegative integer; positive } \\
\text { integer; text }\end{array}$ & $\begin{array}{l}\text { xsd: integer; xsd: nonnegative integer; xsd: positive } \\
\text { integer; string }\end{array}$ & + & + & - \\
\hline verb concept "binary association" & $\begin{array}{l}\text { Object Property, Object Property Domain, Object } \\
\text { Property Range }\end{array}$ & + & + & + \\
\hline partitive verb concept & $\begin{array}{l}\text { Object Property, Object Property Domain, Object } \\
\text { Property Range, SubObject Property Of (partitive } \\
\text { object property) }\end{array}$ & - & $\sim(* 5)$ & + \\
\hline $\begin{array}{l}\text { verb concept "property association" } \\
\text { (together with role) }\end{array}$ & Data Property, Data Property Domain & + & $\sim(* 6)$ & - \\
\hline characteristic (unary verb concept) & $\begin{array}{l}\text { Data Property, Data Property Domain, Data Property } \\
\text { Range "Boolean" }\end{array}$ & - & + & $\sim\left(*^{7}\right)$ \\
\hline
\end{tabular}




\begin{tabular}{|c|c|c|c|c|}
\hline SBVR 1.2 concepts & Our work [15] & $\begin{array}{l}\text { SBVR } 1.2 \\
{[8]}\end{array}$ & $\begin{array}{l}\text { Kendall and } \\
\text { Linehan [12] }\end{array}$ & $\begin{array}{l}\text { Reynares et } \\
\text { al. (SBVR } \\
1.0[13])\end{array}$ \\
\hline $\begin{array}{l}\mathrm{n} \text {-ary verb concept (in this work, } \\
\text { objectified as general concept }+\mathrm{n} \text { verb } \\
\text { concepts) }\end{array}$ & n+1 Class, n Object Properties & - & $+(* 8)$ & - \\
\hline fact & Object Property Assertion | Data Property Assertion & $\sim\left(*^{9}\right)$ & - & - \\
\hline $\begin{array}{l}\text { binary association "concept incorporates } \\
\text { characteristic" }\end{array}$ & $\begin{array}{l}\text { SubClassOf, Data Restriction, Data Exact Cardinality } \\
1\end{array}$ & $\sim(* 9)$ & - & - \\
\hline categorization of general concepts & SubClassOf & $+(* 10)$ & + & + \\
\hline categorization of binary verb concepts & SubPropertyOf & $+(* 11)$ & $\sim(* 12)$ & + \\
\hline concept 1 is coextensive with concept 2 & Equivalent Classes, Equivalent Properties & + & - & - \\
\hline $\begin{array}{l}\text { binary association "is greater than"; "is } \\
\text { less than"; "is equal to": }\end{array}$ & Data Type Restriction & - & - & - \\
\hline $\begin{array}{l}\text { concept (general concept or role) has } \\
\text { extension }\end{array}$ & Object One Of (enumeration of Individuals) & + & - & - \\
\hline segmentation & Equivalent Classes, SubClassOf, Disjoint Union & - & + & + \\
\hline categorization type; characteristic type & Class, which instances are categories or characteristics & - & + & - \\
\hline categorization scheme & Equivalent Classes, SubClassOf & - & + & $+(* 13)$ \\
\hline reference scheme & Has Key & - & - & $-(* 14)$ \\
\hline $\begin{array}{l}\text { universal quantification; existential } \\
\text { quantification }\end{array}$ & All Values From; Some Values From & + & - & + \\
\hline $\begin{array}{l}\text { quantifications "at most n"; "at least n"; } \\
\text { "exactly n"; numeric range } \\
\text { quantification, etc. ( } 6 \text { types) }\end{array}$ & $\begin{array}{l}\text { Max Cardinality n; Min Cardinality n; } \\
\text { Exact Cardinality n;Min Cardinality n, Max } \\
\text { Cardinality m }\end{array}$ & + & $-(* 15)$ & + \\
\hline formal definition of general concept & $\begin{array}{l}\text { Equivalent Classes and axioms, corresponding to the } \\
\text { projection and logical formulations of the definition }\end{array}$ & - & $\sim(* 16)$ & - \\
\hline necessity, obligation & $\begin{array}{l}\text { Axiom, corresponding to the logical formulation of } \\
\text { necessity or obligation formulation }\end{array}$ & - & - & - \\
\hline $\begin{array}{l}\text { impossibility formulation, including } \\
\text { equivalence of classes, properties, or } \\
\text { individuals }\end{array}$ & $\begin{array}{l}\text { Disjoint Classes or Disjoint Properties, or Different } \\
\text { Individuals }\end{array}$ & - & - & - \\
\hline $\begin{array}{l}\text { impossibility formulation, including } \\
\text { logical formulation }\end{array}$ & $\begin{array}{l}\text { complement of the axiom corresponding to the logical } \\
\text { formulation }\end{array}$ & - & - & - \\
\hline instantiation formulation & $\begin{array}{l}\text { Named Individual + Class Assertion (the type of the } \\
\text { Individual) }\end{array}$ & + & - & - \\
\hline conjunction & Intersection Of & + & - & + \\
\hline disjunction & Union Of & + & - & + \\
\hline exclusive disjunction & Disjoint Union & + & - & + \\
\hline equivalence of concepts & $\begin{array}{l}\text { Equivalent Classes, or Equivalent Properties, or Same } \\
\text { Individuals }\end{array}$ & $\sim(* 17)$ & $\sim(* 18)$ & + \\
\hline implication & Only in some cases & - & - & - \\
\hline logical negation of logical formulation & Complement Of & - & - & + \\
\hline $\begin{array}{l}\text { logical negation of equivalence of } \\
\text { general concepts }\end{array}$ & Disjoint Classes & - & - & - \\
\hline logical negation of facts & Negative Property Assertion & - & - & - \\
\hline $\begin{array}{l}\text { logical negation of equivalence of } \\
\text { individual concepts }\end{array}$ & Different Individuals & - & - & - \\
\hline nand formulation & $\begin{array}{l}\text { Expressed through impossibility formulation or logical } \\
\text { negation of conjunction formulation }\end{array}$ & - & - & + \\
\hline nor formulation & $\begin{array}{l}\text { Expressed through impossibility formulation or logical } \\
\text { negation of disjunction formulation }\end{array}$ & - & - & + \\
\hline
\end{tabular}

(*1)Annotations, having range "tagged text"

$(* 2)$ Incorrect (SBVR general concept maps to OWL 2 Class or Data Property)

$\left(*^{3}\right)$ Mapping is not explicit (SBVR role maps to RDF/OWL subject or object)

$\left(*^{4}\right)$ The solution references to our previous work [16], but the purpose of transformation is misunderstood

$\left(*^{5}\right)$ It is assumed that the object property, obtained from a partitive verb concept, cannot have inverse object property what is not true (please see [11])

$\left.{ }^{* 6}\right)$ SBVR property association is specified as a binary verb concept, having more general verb concept "has" instead of concept type "property association")

$\left(*^{7}\right)$ SBVR characteristic is treated as a role what is not true (please see [8])

$\left(*^{8}\right)$ Different solution: SBVR n-ary relation is represented as specified in [38] and objectified as OWL 2 class

$(* 9)$ Mapping is vague

$\left({ }^{* 10}\right)$ From our point of view, OWL 2 Restriction "Disjoint Classes" is too strong for SBVR categorization of general concepts. For this purpose, business rule may be specified, if such a restriction is needed (but often it is not appropriate) 
$\left(*^{11}\right)$ OWL 2 Restriction "Disjoint Properties" is too strong for SBVR categorization of verb concepts (please see $\left.\left({ }^{* 10}\right)\right)$

$(* 12)$ OWL 2 SubProperty Of axioms are considered only for SBVR partitive verb concepts and (incorrectly) property associations but they are appropriate for associations as well

$\left({ }^{* 13}\right)$ Restriction "Disjoint Classes" is too strong for SBVR categorization scheme (please see $\left({ }^{* 10}\right)$ )

$(* 14)$ Incorrect (authors stated that only characteristics are used in reference schemes, but it is not true (please see [8]))

$(* 15)$ SBVR quantification "exactly 1" is incorrectly mapped to OWL 2 Functional Properties, which correspond to quantification "at most 1 "

[11]

$\left({ }^{* 16}\right)$ SBVR formal definition of general concept is limited to several patterns

$(* 17)$ Mapping is limited to Equivalent Properties

$(* 18)$ Limited to one case

\subsection{Transformation of SBVR representations}

The most of attention for transforming SBVR representations to OWL 2 can be found in the publication of Kendall and Linehan [12]. They gave a mapping of SBVR representations including synonyms and synonymous forms, to annotations, having range of tagged text, which can be represented as XML documents. However, their approach has one disadvantage as a random synonymous form is transformed to Inverse Object Property, so the reverse transformation from OWL 2 to SBVR may give a vocabulary different from the original one.

Reynares et al. [13] have not paid attention to SBVR representations. Our SBVR to OWL2 transformation, the $1^{\text {st }}$ version of which was proposed in [16], [17], also is focused on the SBVR meaning. However, we capture the primary representation of every concept in OWL 2 annotation properties "label", "label_sbvr", corresponding to the SBVR representation in the style of written language and in the style of SBVR. One synonymous form is captured for each verb concept wording, which is needed for representing the inverse object property in ontology. All SBVR representations (primary representations, synonyms and synonymous forms) map to Named Individuals of the Lexical Ontology [34]. The Lexical Ontology defines relations between terms, names, and verb concept wordings, and relates them with their meanings. In addition, it includes OWL 2 Object Property for SBVR metamodel association "general concept objectifies verb concept", which allows defining assertions about general concepts that objectify verb concepts (e.g., general concept "price calculation" objectifies verb concept "salesman calculates price"). However, the transformation of SBVR representations is beyond the scope of this research.

Some important SBVR representations (e.g., informal definition of concept, description of vocabulary, note) and concepts having no equivalents in ontology (subject field, speech community) are mapped to OWL 2 annotations. Also, we have extended SBVR with special kinds of notes for specifying ontology IRI and namespace prefix, if needed.

\subsection{Transformation of SBVR extensions introdu- ced for obtaining meaningful OWL 2 ontologies}

We have introduced 15 extensions to SBVR metamodel: 9 verb concepts, 2 binary associations, 2 elementary concepts and 2 representations. The introduced verb concepts can be used as concept types in SBVR vocabulary:

- One synonymous form of binary association or partitive verb concept, specified as verb concept with concept type "inverse verb concept" is transformed to Inverse Object Property of Object Property, corresponding to the primary verb concept wording of the binary association or partitive verb concept;

- 7 verb concepts (e.g., transitive verb concept, "symetric verb concept, etc.) are transformed to Transitive, Symmetric and other characteristics of Object Properties;

- "purely reflexive verb concept" is transformed to class expression "ObjectHasSelf".

These extensions are specified in the SBVR metavocabulary, which is included into other vocabularies used for transforming them into OWL 2 ontologies. Binary associations "is greater than or equal" and "is less than or equal" (similar to SBVR associations "is greater than", "is less than") were introduced for fully expressing Data Type Restrictions.

Finally, "ontology IRI" and "namespace prefix" were introduced as specific types of note for specifying user defined ontology IRI and namespace prefix.

These extensions (except note types) allow representing in SBVR business vocabulary OWL 2 ontology features that are important for inference.

\subsection{Summary of SBVR to OWL 2 transformation comparison}

Table 2 presents the summary of SBVR to OWL 2 transformation comparison. We have found approximately $69 \mathrm{SBVR}$ concepts and propositions worth to transform to OWL 2 ontologies (some rows in Table 1 describe several items). Besides, we have defined requirements and SBVR extensions that allow to represent consistent and full-fledged OWL 2 DL ontologies in SBVR business vocabulary and business rules. It means that we can synchronize business vocabularies and business rules with ontologies, and we can exploit advantages of inference in semantic search, based on SBVR questions in SBVR Structured Language [18]. In addition, our transformation is parameterised and language-independent; it can be adapted to other 
Experimental Investigation of Transformations from SBVR Business Vocabularies and Business Rules to Owl 2 Ontologies

similar languages by setting the defined set of keywords.

In the prototype, the 65 transformations are implemented (51 of 69 theoretically available ones and 14 of 15 transformations available due to SBVR extensions). Part of unimplemented transformations are simple (e.g., various annotations, data types, etc.) or require the extensive work and are not interesting as the subject of research study. The complete implementation of transformations is dependent on the SBVR Structured Language Editor (some concepts were not yet implemented in SBVR SLE). The most complicated concepts are "included vocabularies" and terminological concepts of the SBVR vocabulary, which are the subject of other research works. Finally, implementation of transformations of implications, in general, is possible only with the use of Semantic Web Rules (SWRL), which do not comprise a part of OWL 2 DL but can greatly increase the efficiency of inference. However, currently SWRL rules are not supported in ontology editors as Protégé or TopBraid, and ontology storages as OWLIM. It means that practical applications of SBVR transformations yet require a lot of efforts made by various tools developers. Nevertheless, our transformation covers the greater part of SBVR concepts and allows to obtain almost full variety of OWL 2 DL concepts (except a part of OWL 2 data types, imported ontologies and some non-essential features) in comparison with the most advanced, to our knowledge, related works.

Table 2. Summary of SBVR to OWL 2 transformation comparison

\begin{tabular}{|c|c|c|c|c|}
\hline The comparison criteria & This work & $\begin{array}{l}\text { SBVR 1.2 } \\
\text { specification }\end{array}$ & $\begin{array}{l}\text { Kendall and } \\
\text { Linehan }\end{array}$ & Reynares et al. (SBVR 1.0) \\
\hline The purpose of transformation & $\begin{array}{l}\text { Checking consistency of } \\
\text { SBVR business } \\
\text { vocabularies and } \\
\text { business rules and } \\
\text { transforming them to } \\
\text { ontologies for using in } \\
\text { Semantic Web and } \\
\text { business applications }\end{array}$ & $\begin{array}{l}\text { Establishing } \\
\text { mapping of } \\
\text { concepts }\end{array}$ & $\begin{array}{l}\text { Offering a viable format } \\
\text { for exchanging SBVR } \\
\text { vocabularies between } \\
\text { SBVR tools as an } \\
\text { alternative to XMI }\end{array}$ & $\begin{array}{l}\text { Using of ontology reasoners } \\
\text { to prove the consistency of } \\
\text { business domain information; } \\
\text { generation } \\
\text { of an ontology; implementing } \\
\text { ontologies in software systems }\end{array}$ \\
\hline Coverage of SBVR concepts & $\begin{array}{l}69 \text { of } 69(51 \\
\text { implemented })\end{array}$ & $\begin{array}{l}28 \text { (2 vague, } 3 \\
\text { limited) of } 69\end{array}$ & $\begin{array}{l}41 \text { ( } 2 \text { limited }) \text { of } 69,5 \\
\text { incorrect }\end{array}$ & 29 (1 limited) of 69,3 incorrect \\
\hline SBVR extensions & 15 (14 implemented) & - & 23 & - \\
\hline Coverage of OWL 2 concepts & 74 & 33 & 32 (1 limited) & 34 (4 limited) \\
\hline $\begin{array}{l}\text { Reversibility and no loss of } \\
\text { information }\end{array}$ & + & - & + & - \\
\hline $\begin{array}{l}\text { Requirements for SBVR to obtain } \\
\text { consistent ontologies }\end{array}$ & + & - & - & - \\
\hline $\begin{array}{l}\text { Considering possibility of } \\
\text { inference in ontology }\end{array}$ & + & - & $\begin{array}{l}\text { - (only inverse object } \\
\text { properties) }\end{array}$ & $\begin{array}{l}\text { - (mentioned but not considered } \\
\text { further) }\end{array}$ \\
\hline $\begin{array}{l}\text { Possibility to adapt the } \\
\text { transformation to other languages }\end{array}$ & + & - & - & - \\
\hline $\begin{array}{l}\text { Possibility to set parameters of the } \\
\text { transformation }\end{array}$ & + & - & - & - \\
\hline
\end{tabular}

\section{Experimental approval}

\subsection{Experiment definition}

The goal of the experiment was to evaluate OWL 2 transformation tool prototype with respect to the quality criteria for this research. The evaluation was done from the perspective of users who will use the prototype, and from the perspective of researchers who will analyse the solution, further develop the tool, or propose new ideas.

The following quality criteria are analysed in this experiment:

1) The relevance of SBVR to OWL 2 transformations, executed using the implemented prototype with respect to obtained OWL 2 ontology elements and with respect of preserving information of SBVR elements;

2) The advantages of SBVR extensions, introduced in this work. It will be done by comparing OWL 2 element types obtained from SBVR vocabularies and rules, created using extensions, and without extensions;

3) The possibility to adapt SBVR to OWL 2 transformations to Lithuanian language;

4) The possibility to check the consistency of SBVR vocabulary and rules using OWL 2 reasoners.

\subsection{Quality criteria measures}

We will apply the relevance criteria, which are used in information retrieval and similar fields, and are expressed via the precision and recall. 
The $1^{\text {st }}$ criteria. In this experiment, for measuring relevance of SBVR to OWL 2 transformations with respect to obtained ontology elements, the precision of transformations means the part of OWL 2 elements, obtained during transformation, that are relevant; and recall of transformations means the part of relevant OWL 2 elements obtained during transformation, in comparison with all relevant OWL 2 elements that should be obtained. These criteria can be expressed by formulas:

$$
\begin{aligned}
& P_{T O}=\frac{R T O E \cap A T O E}{A T O E} . \\
& R_{T O}=\frac{R T O E \cap A T O E}{R T O E} . \\
& F_{T O}=2 \times \frac{P_{T O} \times R_{T O}}{P_{T O}+R_{T O}} .
\end{aligned}
$$

Here, $P_{T O}$ is precision of transformations with respect to obtained ontology elements; $R_{T O}$ - recall of transformations with respect to obtained ontology elements; RTOE - relevant transformations to OWL 2 elements; ATOE - executed transformations to OWL 2 elements; $F_{T O}-F$-measure, which means the balanced $F$-score of $P_{T O}$ and $R_{T O}$.

Similarly, the relevance of information preservation with respect to SBVR business vocabulary and business rules during SBVR to OWL 2 transformations can be expressed by formulas:

$$
\begin{aligned}
P_{T S} & =\frac{R T S E \cap A T S E}{A T S E} \\
R_{T S} & =\frac{R T S E \cap A T S E}{R T S E} \\
F_{T S} & =2 \times \frac{P_{T S} \times R_{T S}}{P_{T S}+R_{T S}}
\end{aligned}
$$

Here, $P_{T S}$ is precision of transformations with respect to transformed SBVR elements; $R_{T S}$ - recall of transformations with respect to all SBVR elements that should be transformed; RTSE - relevant transformations of SBVR elements; ATSE - executed transformations of SBVR elements; $F_{T S}-F$-measure with respect to transformed SBVR elements.

The $2^{\text {nd }}$ criteria. Similarly, the advantages of SBVR extensions can be measured by evaluating the part of OWL 2 ontology elements obtained during transformations without SBVR extensions with the part of all OWL 2 ontology elements available during transformations with using SBVR extensions:

$$
\begin{aligned}
P_{T E} & =\frac{R T E \cap A T E}{A T E} . \\
R_{T E} & =\frac{R T E \cap A T E}{R T E} . \\
F_{T E} & =2 \times \frac{P_{T E} \times R_{T E}}{P_{T E}+R_{T E}} .
\end{aligned}
$$

Here, $P_{T E}$ is precision of transformations with respect to types of ontology elements obtained during transformations without using SBVR extensions; $R_{T E}-$ recall of transformations without using SBVR extensions; RTE - relevant transformations of OWL 2 elements due to SBVR extensions (i.e., RTE coincides with $R T O E$ in formulas (1), (2); ATE - executed transformations to OWL 2 elements without using
SBVR extensions; $F_{T E}-F$-measure with respect to ontology elements obtained without using SBVR extensions in comparison with relevant elements that should be obtained due to SBVR extensions.

The percent of increasing a number of OWL 2 element types due to SBVR extensions POTE is evaluated by formula:

$$
P_{\text {OTE }}=\frac{\text { OTE }_{U}-\text { OTE }}{\text { OTE }} \times 100 \% \text {. }
$$

Here, OTE is the number of OWL 2 element types, available to obtain from a certain SBVR vocabulary without using SBVR extensions created in this work, and $O T E_{U}$ is the number of OWL 2 element types obtained using SBVR extensions.

The $3^{\text {rd }}$ criteria for evaluating the possibility to adapt SBVR to OWL 2 transformations to Lithuanian language will be evaluated by comparing relevance of transformations of Lithuanian and English ontologies evaluated by formulas (1)-(10).

The $4^{\text {th }}$ criteria will be evaluated by checking consistency of ontologies obtained during transformations, and making improvements in SBVR business vocabularies and business rules in cases of inconsistencies found. The number of instances $N I_{i}$ of investigated ontologies and number of inconsistencies $N S_{i}$ from SBVR business vocabularies and business rules removed will be given for validating the fulfilment of the $4^{\text {th }}$ criterion. As the formal procedure for obtaining a sufficient number of instances [26] is a very labour intensive process for investigating 9 selected SBVR vocabularies, types of dependencies among ontology concepts were analysed and the following rules were established:

- All created individuals must be different except individuals for satisfying assertions of equivalence of individuals;

- At least one individual must be created for each most specific primitive (non-derivable) class, as individuals of subclasses also are individuals of their superordinate classes, and individuals of derivable classes are derived from individuals of primitive classes;

- At least one assertion must be created for each non-derivable most-specific data property and object property (e.g., one assertion can be enough for inverse object properties);

- At least 2 correct assertions must be created for checking transitive object properties and 2 incorrect assertions must be created for checking irreflexive and asymmetric object properties (incorrect assertions after checking should be removed from ontology model);

- At least $n$ individuals must be created for classes, which are ranges of object properties having "minimum $n$ ", "exactly $n$ " cardinality restrictions for domain classes of these object properties;

- At least $n$ different individuals and $m$ property assertions must be created for satisfying each axiom (which depends on $n$ individuals and $m$ 
Experimental Investigation of Transformations from SBVR Business Vocabularies and Business Rules to Owl 2 Ontologies

property assertions), supporting derivation of individuals of derivable classes;

- At least $n$ different incorrect individuals and $m$ property assertions must be created for checking each axiom (which depends on $\mathrm{n}$ individuals and $m$ property assertions), specifying ontology consistency constraints; after checking, incorrect individuals and assertions must be removed from the ontology model.

Creation of ontology model can be executed as an iterative process consisting of consequent steps; in each step, the consistency of ontology is checked and ontology class and property views are explored using Protégé editor until the ontology model becomes complete.

Experiment context was SBVR SLE editor with integrated SBVR to OWL 2 transformation tool prototype. Experiment was executed by the first author of this paper in the local computer using SBVR SLE for defining 9 SBVR business vocabularies and business rules for various domains. Protégé ontology editor was used for making the inference and checking consistency of the ontologies, received during transformation.

For checking the consistency of business rules, OWL 2 ontology was revised in Protégé 4.3 and checked with Hermit 1.3.8 reasoner. For checking consistency of ontology, the sufficient set of individuals and property assertions was created in accordance with principles described for evaluating the $4^{\text {th }}$ criterion. The following SBVR business vocabularies and business rules were analysed (all vocabularies, rules and obtained ontologies are available at http://s2o.isd.ktu.lt/ [36]):

- The representative example of SBVR business vocabulary and business rules for Photo Equipment domain (Photo Eq.) in SBVR Structured English language, presented in [15], which covers SBVR concepts and business rules for validating each type of transformation;

- Domain specific vocabularies of SemantikaLT project in SBVR Structured Lithuanian language for verifying applicability of transformations to Lithuanian language;

- Loans vocabulary, which was created regardless of the requirements and extensions, which were introduced for development of vocabularies, dedicated for creating ontologies.

\subsection{Experiment results}

Results for measuring relevance of SBVR to OWL 2 transformations with respect to obtained ontology elements (formulas (1)-(3)) are presented in Table 3.

As it is seen from Table 3, all implemented transformations are relevant; no problems arise with transformation of vocabularies in Lithuanian language or with vocabulary, which was created regardless of the requirements and extensions, which were introduced for development of vocabularies, dedicated for creating ontologies. However, the expressivity of ontologies, obtained during transformations, is less when SBVR extensions are not used. The SBVR vocabulary for Photo Equipment domain has a rich set of SBVR elements and extensions; other vocabularies were much simpler. These aspects are reflected in the following evaluations of other criteria.

Table 3. Evaluation of relevance of SBVR to OWL 2 transformations with respect to obtained ontology

\begin{tabular}{lccccc}
\hline SBVR voc. & ATOE & $\boldsymbol{R T O E}$ & $\boldsymbol{P}_{\boldsymbol{T O}}$ & $\boldsymbol{R}_{\boldsymbol{T O} \boldsymbol{O}}$ & $\boldsymbol{F}_{\boldsymbol{T O} \boldsymbol{O}}$ \\
\hline 1. Photo Eq. & 555 & 555 & 1 & 1 & 1 \\
2. SemLT & 72 & 72 & 1 & 1 & 1 \\
3. Locations & 197 & 197 & 1 & 1 & 1 \\
4. Agents & 151 & 151 & 1 & 1 & 1 \\
5. Events & 653 & 653 & 1 & 1 & 1 \\
6. Politics & 1103 & 1103 & 1 & 1 & 1 \\
7. Business & 1327 & 1327 & 1 & 1 & 1 \\
8. Public sector & 1357 & 1357 & 1 & 1 & 1 \\
9. Loans & 762 & 762 & 1 & 1 & 1 \\
\hline
\end{tabular}

The results of evaluation of relevance of information preservation with respect to SBVR business vocabulary and business rules during SBVR to OWL 2 transformations (formulas (4)-(6)) are presented in Table 4.

Table 4. Evaluation of relevance of information preservation with respect to SBVR elements during SBVR to OWL 2 transformations

\begin{tabular}{lccccc}
\hline SBVR voc. & $\boldsymbol{A T S E}$ & $\boldsymbol{R T S E}$ & $\boldsymbol{P}_{\boldsymbol{T S}}$ & $\boldsymbol{R}_{\boldsymbol{T S}}$ & $\boldsymbol{F}_{\boldsymbol{T S}}$ \\
\hline 1. Photo Eq. & 555 & 556 & 1 & 0,998 & 0,999 \\
2. SemLT & 72 & 72 & 1 & 1,000 & 1,000 \\
3. Locations & 197 & 340 & 1 & 0,597 & 0,734 \\
4. Agents & 151 & 460 & 1 & 0.328 & 0,494 \\
5. Events & 653 & 731 & 1 & 0,893 & 0,944 \\
6. Politics & 1103 & 1814 & 1 & 0.576 & 0.731 \\
7. Business & 1327 & 2014 & 1 & 0,659 & 0,794 \\
8. Public s. & 1357 & 2253 & 1 & 0.602 & 0,752 \\
9. Loans & 762 & 764 & 1 & 0,997 & 0,999 \\
Average & & & 1 & 0,857 & 0,840 \\
Standard deviation & & 0 & 0,183 & 0,180 \\
\hline
\end{tabular}

As it is seen from experiment results, the relevance of preserving the information of SBVR vocabularies and business rules is less for Lithuanian vocabularies. This fact can be explained by the presence of nontransformable elements in Lithuanian SBVR vocabularies and business rules. Such elements were synonyms and synonymous forms, which were included in Lithuanian SBVR vocabularies (as was decided, synonyms and synonymous forms should be transformed into separate lexical ontologies and such transformations are beyond the scope of this work).

The results of evaluating the impact of non-use of SBVR extensions in SBVR to OWL 2 transformations (formulas (7)-(9)) are presented in Table 5. Here ATE 
is a number of OWL 2 elements obtained in transformations without using SBVR extensions. As it is seen from comparison of ATE with ATOE in Table 3, SBVR extensions allow expressing more OWL 2 elements. Therefore, the recall $R_{T E}$ in Table 5 is obviously less than recall $R_{T O}$ in Table 3 .

Table 5. Evaluation of impact of non-use of SBVR extensions for SBVR to OWL 2 transformations

\begin{tabular}{lccccc}
\hline SBVR voc. & $\boldsymbol{A T E}$ & $\boldsymbol{R T E}$ & $\boldsymbol{P}_{\boldsymbol{T E}}$ & $\boldsymbol{R}_{\boldsymbol{T E}}$ & $\boldsymbol{F}_{\boldsymbol{T E}}$ \\
\hline 1. Photo Eq. & 425 & 555 & 1 & 0,766 & 0,867 \\
2. SemLT & 42 & 72 & 1 & 0,583 & 0,737 \\
3. Locations & 127 & 197 & 1 & 0,645 & 0,784 \\
4. Agents & 97 & 151 & 1 & 0,642 & 0,782 \\
5. Events & 337 & 653 & 1 & 0,516 & 0,681 \\
6. Politics & 599 & 1103 & 1 & 0,543 & 0,704 \\
7. Business & 727 & 1327 & 1 & 0,548 & 0,708 \\
8. Public sector & 801 & 1357 & 1 & 0,590 & 0,742 \\
9. Loans & 534 & 762 & 1 & 0,700 & 0,824 \\
Average & & & 1 & 0,615 & 0,759 \\
Standard deviation & & & 0 & 0,081 & 0,061 \\
\hline
\end{tabular}

The percent $P_{\text {OTE }}$ (calculated according formula (10)) of increasing a number of OWL 2 element types due to SBVR extensions is presented in Table 6. The percent $P_{\text {OTE }}$ for investigated ontologies has varied from $14 \%$ to $20 \%$ for the cases when extensions were used. For Loan contracts vocabulary, the SBVR extensions were not used at all, but the Loan business vocabulary and business rules were more complex. So the number of OWL 2 element types was greater in comparison with ontologies, obtained from simpler business vocabularies. The greatest values of increasing the percent $P_{\text {OTE }}$ of obtained OWL 2 element types is $20 \%$ for Photo Equipment vocabulary where all extensions were used. The remaining vocabularies were much simpler and have used the less number of SBVR extensions. However, creation of complex ontologies would require using all SBVR extensions, created in this research.

Table 6. Evaluation of increasing the number of OWL 2 element types due to SBVR extensions

\begin{tabular}{lcccc}
\hline $\begin{array}{l}\text { SBVR } \\
\text { vocabulary }\end{array}$ & $\begin{array}{c}\text { SBVR } \\
\text { extensions } \\
\text { used }\end{array}$ & OTE $_{\boldsymbol{U}}$ & OTE & $\begin{array}{c}\text { PoTE } \\
\text { (\%) }\end{array}$ \\
\hline 1. Photo Eq. & 13 & 65 & 52 & 20 \\
2. SemLT & 2 & 12 & 10 & 17 \\
3. Locations & 2 & 12 & 10 & 17 \\
4. Agents & 2 & 12 & 10 & 17 \\
5. Events & 3 & 17 & 14 & 18 \\
6. Politics & 3 & 21 & 18 & 14 \\
7. Business & 3 & 21 & 18 & 14 \\
8. Public sector & 3 & 21 & 18 & 14 \\
9. Loans & 0 & 22 & 22 & 0 \\
\hline
\end{tabular}

As it is seen from experiment results, precision of SBVR to OWL 2 transformations with respect to ob- tained OWL 2 elements is evaluated to 1 for both languages. The recall of SBVR to OWL 2 transformations with respect to transformed SBVR elements is slightly worse for Lithuanian language, but this depends on the fact that analysed Lithuanian vocabularies had more synonyms and synonymous forms, whose transfor-mations to OWL 2 were not realized in this research.

Table 7. Evaluation of possibility to adapt SBVR to OWL 2 transformations to Lithuanian language

\begin{tabular}{lcc}
\hline Criteria & $\begin{array}{c}\text { Structured } \\
\text { English (1,9) }\end{array}$ & $\begin{array}{c}\text { Structured } \\
\text { Lithuanian (2-8) }\end{array}$ \\
\hline Average $A T O E$ & 659 & 694 \\
Average $R T O E$ & 659 & 694 \\
Average $P_{T O}$ & 1 & 1 \\
Average $R_{T O}$ & 1 & 1 \\
Average $F_{T O}$ & 1 & 1 \\
Average ATSE & 660 & 1098 \\
Average RTSE & 659 & 694 \\
Average $P_{T S}$ & 1 & 1 \\
Average $R_{T S}$ & 0,998 & 0,787 \\
Average $F_{T S}$ & 0,999 & 0,786 \\
Average standard & 0 & 0 \\
deviation of $P_{T S}$ & & 0,191 \\
$\begin{array}{l}\text { Average standard } \\
\text { deviation of } R_{T S}\end{array}$ & 0,001 & 0,179 \\
$\begin{array}{l}\text { Average standard } \\
\text { deviation of } F_{T S}\end{array}$ & 0 & \\
\hline
\end{tabular}

The results of evaluating the possibility to check the consistency of SBVR vocabulary and rules using OWL 2 reasoners are presented in Table 8 .

Table 8. Evaluation of possibility to check the consistency of SBVR business vocabulary and business rules using OWL 2 reasoners

\begin{tabular}{lccc}
\hline $\begin{array}{l}\text { SBVR } \\
\text { vocabulary }\end{array}$ & $\begin{array}{c}\text { Number of } \\
\text { individuals }\end{array}$ & $\begin{array}{c}\text { Number of } \\
\text { assertions }\end{array}$ & $\begin{array}{c}\text { Corrected } \\
\text { elements }\end{array}$ \\
\hline 1. Photo Eq. & 41 & 43 & 20 \\
2. SemLT & 10 & 13 & 0 \\
3. Locations & 21 & 20 & 5 \\
4. Agents & 15 & 16 & 3 \\
5. Events & 44 & 70 & 12 \\
6. Politics & 68 & 81 & 14 \\
7. Business & 81 & 91 & 25 \\
8. Public sector & 88 & 93 & 26 \\
9. Loans & 15 & 31 & 1 \\
\hline
\end{tabular}

This part of experiment has shown that transforming SBVR business vocabularies and business rules to OWL 2 ontologies is useful even if ontologies are not needed for other purpose (e.g., semantic search). The transformations help to find errors and inconsistencies in SBVR business vocabularies and business rules, and to correct them. 
Experimental Investigation of Transformations from SBVR Business Vocabularies and Business Rules to Owl 2 Ontologies

\subsection{Threats to validity}

The possible threats to validity of SBVR to OWL 2 transformations were examined.

Internal validity could be damaged by instrumentation. The confounding factor that makes influence on internal validity of experimental results is dependency of the implementation of SBVR to OWL 2 transformations on the SBVR Structured Language Editor. For eliminating this threat, the development of the transformation component and SBVR SLE has been coordinated with each other. The future evolution of SBVR SLE can both provide opportunities to improve the SBVR to OWL 2 transformations or cause problems for their correctness.

External validity. The advantages of SBVR to OWL 2 transformations can be generalized to SBVR vocabularies and rules, which meet the requirements and use SBVR extensions for OWL 2 defined in this work.

Such requirements may seem like an obstacle to free development of business vocabularies. In fact, requirements for vocabulary and rules do not cause a threat. By contraries, these requirements correspond to methodology and good practise of conceptual modelling; disregarding those requirements means that the quality of the vocabulary is poor and it is unusable in real life situations.

For analysing the threat of unused SBVR extensions, the experiment was conducted with various vocabularies and rule sets; part of them was created without using all of the proposed SBVR extensions, or without an intention to obtain ontology. It can be seen that disregarded extensions decrease the expressivity of ontologies, but do not affect effectiveness of transformations.

In order to exploit all advantages of SBVR to OWL 2 transformations in a broader context, methodology and requirements for creating SBVR business vocabulary and business rules must be carefully specified and explained by examples. Templates for constructing typical SBVR concepts, sets of concepts and rules must be presented together with explanation of undesirable issues in the case of deviation from such requirements. In order to achieve the potential benefits of semantic technologies, the society has to pay the proper attention to development of semantic technologies; the required possibilities have to be clarified and supported by tools, and then they will find a use in business practice.

\section{Conclusions and future works}

The paper presents analysis and evaluation of the proposed SBVR to OWL 2 transformation according to the established criteria: the transformation was aimed to be model driven, lossless and reversible; covering transformable SBVR concepts and OWL 2 DL concepts, important for inference, and supporting the consistency and normalization of resulting ontology. The detailed comparison of the proposed solution with the most advanced related works was made and the experiment was conducted for investigating the implemented transformation prototype with nine SBVR business vocabularies and business rule sets.

The novelty of the work can be described by the following results:

1) Defined SBVR extensions, specified in SBVR metavocabulary for transformation to OWL 2, and the comprehensive usage of SBVR concepts allow to cover richer subsets of SBVR and OWL 2 metamodels in SBVR to OWL 2 transformations in comparison with other existing works.

2) The selected subset of SBVR metamodel concepts with extensions, introduced in this work, can represent full-fledged OWL 2 DL ontologies, enabling ontology creation from SBVR business vocabulary and rules described in SBVR structured natural language understandable for business participants, analysts, software developers and even end users.

3) The defined set of requirements for SBVR business vocabularies and business rules allow transforming them to the consistent OWL 2 DL ontologies.

4) Transformation works for SBVR business vocabularies and rules in Lithuanian and English languages, and can be adapted to other similar languages by adjusting the defined set of keywords.

To our knowledge, the previous related works have not considered the coverage of OWL 2 DL concepts, consistency of obtained ontologies, and possibility to adapt transformations for SBVR business vocabularies and rules described in different natural languages. SBVR extensions, specified in the metavocabulary, allow extending SBVR knowledge model without changing the original SBVR metamodel.

\section{Acknowledgements}

The work is supported by the project VP1-3.1ŠMM-10-V-02-008 „Integration of Business Processes and Business Rules on the Basis of Business Semantics" (2013-2015), which is funded by the European Social Fund (ESF).

\section{References}

[1] T. Halpin. Verbalizing Business Rules. http://www.orm.net.

[2] M. Jarrar, M. Keet, P. Dongilli. Multilingual verbalization of ORM conceptual models and axiomatized ontologies. STARLab Technical Report. Vrije Universiteit Brussel, 2006.

[3] P. Kaplanski. Modeling Object Oriented Systems via Controlled English Verbalization of Description Logic. In: Second Workshop on Controlled Natural Languages CNL 2010, CEUR Workshop proceedings 622, 2010, pp. 1-5.

[4] K. Kaljurand. Attempto Controlled English as a Semantic Web Language. Doctoral thesis, Dec. 2007. University of Tartu, 2007. 
[5] R. Schwitter, K. Kaljurand, A. Cregan, C. Dolbear, G. Hart. A Comparison of three Controlled Natural Languages for OWL 1.1. In: OWL: Experiences and Directions (OWLED 2008), 2008, 1-10.

[6] T. Kuhn. A Survey and Classification of Controlled Natural Languages. Computational Linguistics, 2014, Vol. 40, No. 1, 121-170.

[7] OMG, 2008. Semantics of Business Vocabulary and Business Rules (SBVR), Version 1.0. OMG Document Number: formal/2008-01-02, 2008.

[8] OMG, 2013. Semantics of Business Vocabulary and Business Rules (SBVR), Version 1.2. OMG Document Number: formal/2013-11-05, 2013.

[9] T. Halpin. ORM 2 Graphical Notation. Technical Report ORM2-01, Neumont University, 2005.

[10] T. Halpin, T. Morgan. Information Modeling and Relational Databases. Elsevier Inc., 2008.

[11] W3C, 2012a. OWL 2 Web Ontology Language Structural Specification and Functional-Style Syntax (Second Edition). W3C Recommendation 11 December 2012.

[12] E. Kendall, M.H. Linehan. Mapping SBVR to OWL2. IBM Research Report, RC25363 (WAT1303-040) March 16, 2013.

[13] E. Reynares, M.L. Caliusco, M.R. Galli. An Automatable Approach for SBVR to OWL 2 Mappings. In: XVI Ibero-American Conference on Software Engineering CIbSE 2013, Montevideo, Uruguay, 2013, pp. 201-214.

[14] E. Reynares, M.L. Caliusco, M.R. Galli. Approaching the feasibility of SBVR as modeling language for ontology development: An exploratory experiment. Expert Systems with Applications, 2014, Vol. 41, No. 4, 1576-1583.

[15] J. Karpovič, G. Kriščiūnienè, L. Ablonskis, L. Nemuraitè. The comprehensive mapping of semantics of business vocabulary and business rules (SBVR) to OWL 2 ontologies. Information Technology and Control, 2014, Vol. 43, No. 3, 289-302.

[16] J. Karpovic, L. Nemuraite. Transforming SBVR business semantics into Web ontology language OWL2: main concepts. In: Information Technologies' 2011: Proceedings of the 17th international conference on Information and Software Technologies, IT 2011, Kaunas, Lithuania, April 27-29, 2011, pp. 231-238.

[17] J. Karpovic, L. Nemuraite, M. Stankeviciene. Requirements for semantic business vocabularies and rules for transforming them into consistent OWL2 ontologies. In: Information and software technologies: 18th International Conference, ICIST 2012, Kaunas, Lithuania, September 13-14, 2012. 319, Berlin, Heidelberg: Springer, 2012, pp. 420-435.

[18] A. Šukys, L. Nemuraitė, E. Šinkevičius, B. Paradauskas. Querying ontologies on the base of semantics of business vocabularies and business rules. In: Information Technologies' 2011 : Proceedings of the 17th international conference on Information and Software Technologies, IT 2011, Kaunas, Lithuania, April 27-29, 2011, pp. 247-254.

[19] G. Guizzardi, G. Wagner, N. Guarino, M. Sinderen. An Ontologically Well-Founded Profile for UML Conceptual Models. In: A. Person and J. Stirna (Eds.): CAISE 2004, LNCS, Vol. 3084, 2004, pp. 112-126.

[20] T.R. Gruber. Toward Principles for the Design of Ontologies Used for Knowledge Sharing. Technical report, KSL-93-04. Knowledge Systems Laboratory, Stanford University, 1993.

[21] Grüninger, M., Fox, M. S. Methodology for the Design and Evaluation of Ontologies. In: Proceedings of the IJCAI Workshop on Basic Ontological Issues in Knowledge Sharing. Menlo Park Calif.: AAAI Press, 1995, pp. 1-10.

[22] A.L. Rector. Normalisation of ontology implementations: Towards modularity, re-use, and maintainability. In: Proceedings Workshop on Ontologies for Multiagent Systems (OMAS) in conjunction with European Knowledge Acquisition Workshop, 2002, pp. 1-16.

[23] A.L. Rector. Modularisation of domain ontologies implemented in description logics and related formalisms including owl. In: Genari, J. (Ed.): Proceedings of the 2nd International Conference on Knowledge Capture $K-C A P$ 03, ACM, 2003, 121-128.

[24] L. Nemuraité, B. Paradauskas. A methodology for engineering OWL 2 ontologies in practise considering their semantic normalisation and completeness. Electronics and Electrical Engineering, 2012, Vol. 4, No. 120, 89-94.

[25] E. Vyšniauskas, L. Nemuraitė, R. Butleris, B. Paradauskas. Reversible lossless transformation from OWL 2 ontologies into relational databases. Information Technology and Control, 2011, Vol. 40, No. 4, 293-306.

[26] E. Vyšniauskas, L. Nemuraitė, B. Paradauskas. Preserving semantics of OWL 2 ontologies in relational databases using hybrid approach. Information Technology and Control, 2012, Vol. 41, No. 2, 103-115.

[27] F. Jouault, F. Allilaire, J. Bézivin, I. Kurtev. ATL: a QVT-like transformation language. In: Companion to the 21st ACM SIGPLAN symposium on Objectoriented programming systems, languages, and applications (OOPSLA '06), ACM New York, NY, USA, 2006, pp. 719-720.

[28] H. Ehrig, K. Ehrig, C. Ermel, F. Hermann, G. Taentzer. Information preserving bidirectional model transformations. In: Proceedings of Fundamental Approaches to Software Engineering (FASE 2007), LNCS 4422, 2007, Springer, Heidelberg, pp. 72-86.

[29] P. Stevens. Bidirectional model transformations in QVT: semantic issues and open questions. Software and Systems Modeling, 9, 2010, pp. 7-20.

[30] J.L. Hainaut, C. Tonneau, M. Joris, M. Chandelon. Transformation-based Database Reverse Engineering. In: Proceedings of the 12th International Conference on the Entity-Relationship Approach, LNCS, 823, Springer-Verlag, 1993, pp. 364-375.

[31] G. Bernotaityte, L. Nemuraite, R. Butkiene, B. Paradauskas. Developing SBVR vocabularies and business rules from OWL2 ontologies. In: Information and Software Technologies: 19th international conference, ICIST 2013, Kaunas, Lithuania, October 10-11, 2013. CCIS 403, Springer: Berlin, Heidelberg, 2013, pp. 134-145.

[32] W3C 2012b. B. Motik, P.F. Patel-Shnaider, B.C. Grau (Eds.) OWL 2 Web Ontology Language Direct Semantics (Second Edition). W3C Recommendation 11 December 2012.

[33] E. Sirin, B. Parsia, B.C. Grau, A. Kalyanpur, Y. Katz. Pellet: A Practical OWL-DL Reasoner. Web 
Experimental Investigation of Transformations from SBVR Business Vocabularies and Business Rules to Owl 2 Ontologies

Semantics: Science, Services and Agents on the World Wide Web, 2007, Vol. 5, No. 2, 51-53.

[34] G. Krisciuniene, L. Nemuraite, R. Butkiene, B. Paradauskas. Lexical ontology model based on SBVR representations. International Journal of Advances in Computer Science \& Its Applications, 2014, Vol. 4, No. 3, 103-107.

[35] OMG, 2011. Date-Time Vocabulary (Date-Time). Version 1.0. OMG Document bmi/2011-08-01.

[36] J. Karpovic, A. Sukys, L. Nemuraite. s2o: SBVR to OWL 2 Online Converter. May 15, 2014. http://s2o.isd.ktu.lt/.

[37] SemantikaLT. Syntactic-semantic analysis and search system for Lithuanian Internet, corpus and public sector applications (2012-2014). No VP2-3.1-IVPK12-K, 2014

[38] W3C, 2006. Defining N-ary Relations on the Semantic Web. W3C Working Group Note. http://www.w3.org/TR/swbp-n-aryRelations/ .

[39] W3C, 2012c. P.F. Patel-Schneider (Ed.) OWL $2 \mathrm{Web}$ Ontology Language RDF-Based Semantics (Second Edition). W3C Recommendation 11 December 2012.

[40] W3C, 2012d. M. Schneider, B. Motik (Eds.) $O W L 2$ Web Ontology Language Mapping to RDF Graphs. W3C Recommendation 11 December 2012.

[41] The University of Manchester. OWL Syntax Converter. http://owl.cs.manchester.ac.uk/converter/.

Received December 2014. 